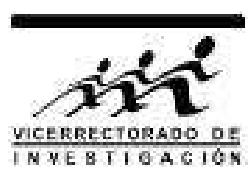

\title{
Estructura electrónica en sistemas cristalinos de Ge, GaP y SiC
}

\author{
César Cabrera ${ }^{* 1,2}$ y Máximo Poma ${ }^{1}$ \\ ${ }^{1}$ Universidad Nacional Mayor de San Marcos, Ap. Postal 14-0149, Lima, Perú \\ ${ }^{2}$ Universidad Nacional del Callao, Av. Juan Pablo II S/N, Bella-Vista, Callao, Perú
}

Recibido 07 diciembre 2017 - Aceptado 29 enero 2018

Se estudia la estructura electrónica de los cristales de Germanio y de los compuestos cristalinos de GalioFosforo y de Silicio-Carbón, usando un potencial funcional de la densidad local de spin (LDA) y el método LMTO se calculan las bandas de energía, la banda de energía prohibida, la densidad de estados DOS, la energía total del sistema cristalino. Las propiedades electrónicas se ajustan bien a los resultados experimentales.

Palabras claves: bandas de energía, densidad de estados, red cristalina.

\section{Electronic Structure in crystalline system of $\mathrm{Ge}, \mathrm{GaP}$ and $\mathrm{SiC}$}

Be study the electronic structure of $\mathrm{Ge}$ and of the compounds $\mathrm{GaP}$ and $\mathrm{SiC}$, we utilized a local density potential (LDA) and the method (LMTO) we present the calculated energy band, band-gaps, the density occupied states DOS, the total energy of crystalline system. There is good agreement between the calculated electronic properties and experimental results.

Keywords: energy bands, Density of States, crystal lattice.

\section{Introducción}

Debido a sus propiedades electrónicas, mecánicas y químicas a los semiconductores como el Germanio, GalioFósforo y Silicio-Carbón, los han convertido en los materiales más prometedores para sus aplicaciones en dispositivos de alta temperatura y potencia. La gran energía de enlace de estos materiales los hace resistentes a los ataques químicos y a la exposición de radiación solar.

De cualquier modo, en muchos cálculos LDA para semiconductores o aislantes la brecha de la energía prohibida se desestima entre unos treinta a cincuenta por ciento. Este problema de la banda de energía prohibida esta relacionado a una baja energía de la banda de conducción en muchos cálculos LDA de computación y no se ajustan bien a los resultados experimentales. De esta manera en este artículo presentamos una alternativa para el cálculo de la estructura electrónica de sólidos cristalinos.

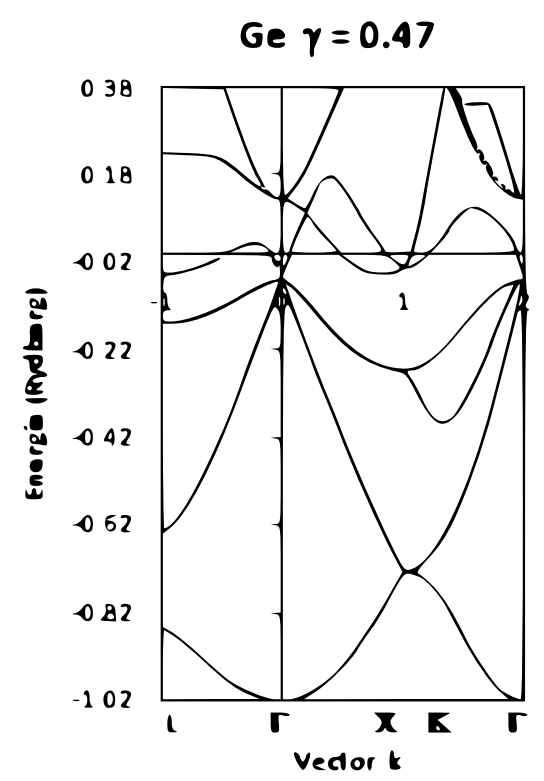

Figura 1: Bandas de energía del cristal de Germanio

*ccabreraa@unmsm.edu.pe 


\section{Fundamento teórico}

En este artículo, reportamos el cálculo ab-initio de la estructura electrónica (las bandas de energía, la densidad de estados DOS) y la energía total en el estado fundamental del Germanio y de los compuestos binarios de Galiofósforo GaP [1] y del Silicio-Carbón SiC [2], usando el método de los orbitales lineales muffin-tin en la aproximación de las esferas atómicas LMTO-ASA [3], modelo que usa la teoría del funcional densidad (DFT) [4] en su forma local.

Como potencial de interacción electrónica, que actúa sobre los electrones en la red cristalina, usamos un potencial efectivo de tipo Muffin-Tin. Este potencial, que formulamos utilizando el funcional densidad de spin local, usa como aproximación para el término de intercambio y correlación, el potencial de correlación para un gas homogéneo de electrones de Ceperley-Alder discutido excelentemente por MacLaren [5] en 1991. Este potencial de interacción ya contiene toda la información de la estructura cristalina, como la constante de la red cristalina, la posición de los átomos en la celda convencional y se calcula a partir de la densidad electrónica del átomo libre pero con una distribución diferente para la red cristalina del sólido [6].

$$
\text { GaP } \gamma=0.0
$$

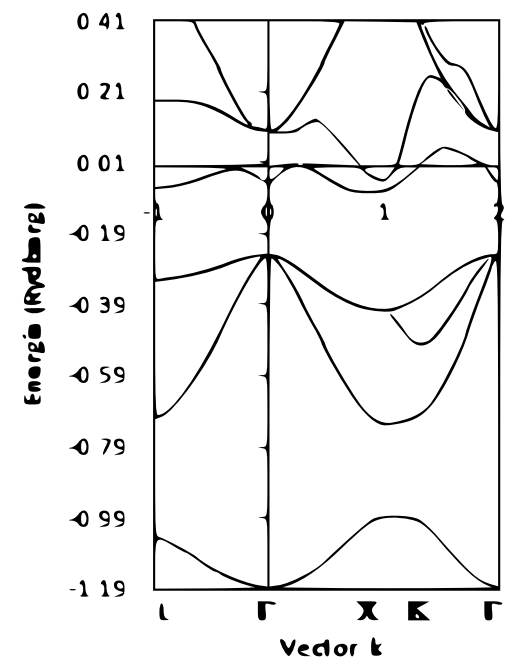

Figura 2: Bandas de energía del cristal binario de Galio-Fosforo

Con este potencial, se resuelve la ecuación autoconsistente de Kohn-Sham [7], para un electrón que interacciona con el potencial efectivo de la red cristalina, usando el método LMTO de Hans L. Skriver [3, 6] y se calculan los parámetros potenciales que nos permite formular la base LMTO ortogonal que se usa para calcular la estructura de las bandas de energía y calcular la densidad de estados DOS. Finalmente con la DOS ya conocida, se calcula la energía total del sólido cristalino.

$$
\text { Sic } \gamma=0.0
$$

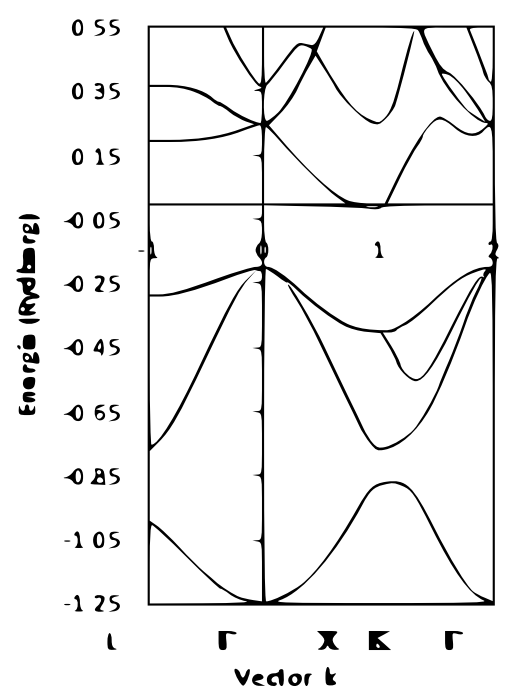

Figura 3: Bandas de energía del cristal binario de SilicioCarbón

\section{Datos}

Para calcular el potencial efectivo que usamos para resolver la ecuación de Kohn-Sham para el sólido, usamos la constante de red de 10.696 au para $\mathrm{Ge}$, de 10.299 au para $\mathrm{GaP}$ y de 8.220 au para $\mathrm{SiC}$ [8]. La tabla [1] muestra el radio de las esferas atómicas que usamos para el cálculo del potencial efectivo. Para calcular el radio de la esfera atómica del Galio y del fósforo ambos de red cristalina ortorrombica, usamos las constantes de la red publicada N.W. Ashcroft [9].

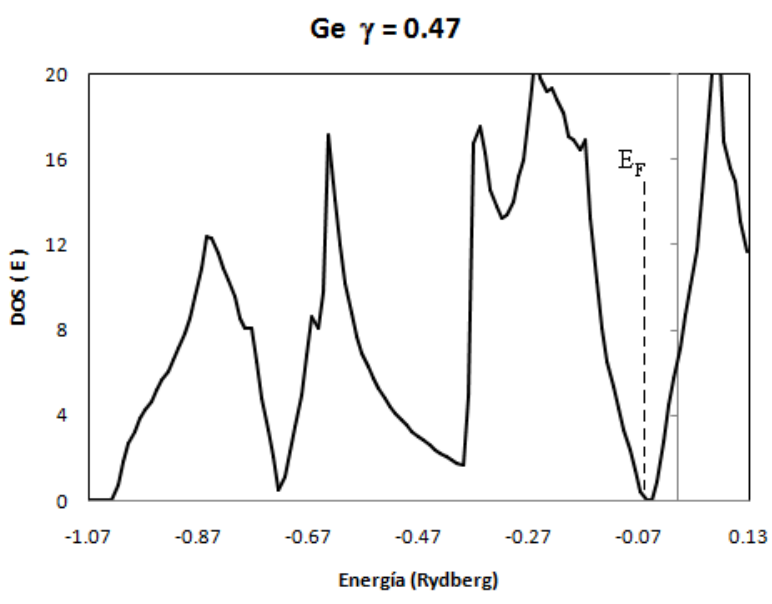

Figura 4: Densidad de estados del cristal de Ge. 


\section{Resultados y discusión}

Las propiedades electrónicas de Ge obtenidas para un cálculo LDA con un valor del parámetro de transferencia de carga electrónica a la red cristalina, de 0.47, resultan muy buenas, puesto que la estructura de las bandas de energía calculadas aquí, presenta un gap y el típico perfil de las bandas de energía por ejemplo, reportadas por Wang en 1993 [10]. Sin embargo, entre la banda de valencia y la banda de conducción calculadas para germanio aquí, presenta un pequeño gap indirecto de $0.312 \mathrm{eV}$. Este brecha de energía prohibida se aprecia en la Fig.[1] que muestra una gráfica de las bandas de energía en las direcciones de alta simetría para la red cristalina del Ge.

\section{GaP $\gamma=\mathbf{0 . 0}$}

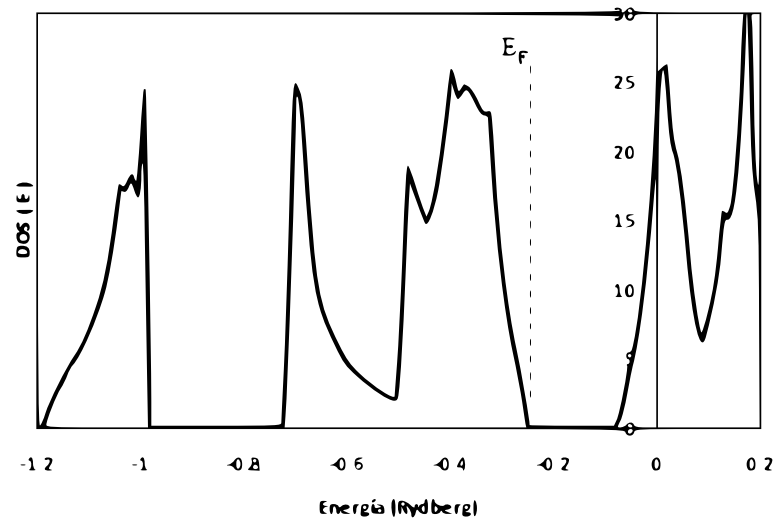

Figura 5: Densidad de estados del cristal binario de GalioFosforo

$$
\text { SiC } \gamma=0.0
$$

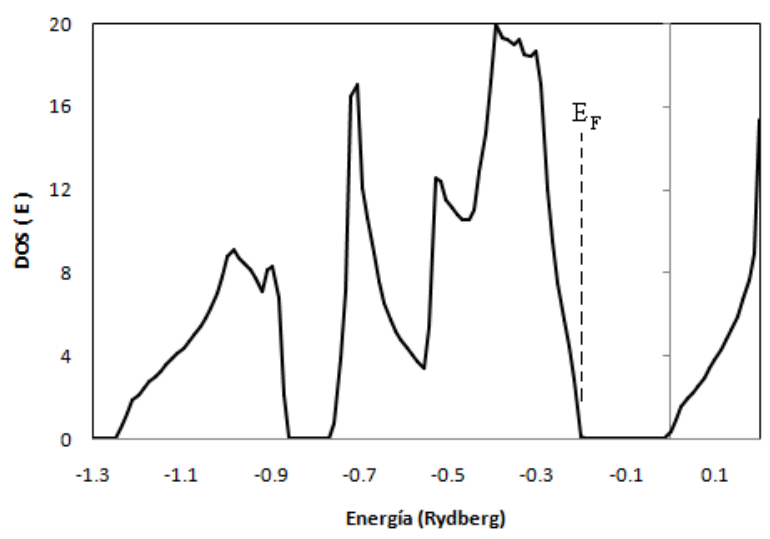

Figura 6: Densidad de estados del cristal binario de SilicioCarbón

Para el caso de compuestos binarios con estructura cristalina blenda de cinc el $\mathrm{GaP}$ y el $\mathrm{SiC}$, en ambos sistemas cristalinos, los resultados del calculo LDA presentan excelentes propiedades electrónicas para el valor de 0.0 , del parámetro de transferencia de carga electrónica a la red cristalina. Las bandas de energía de los dos compuestos $\mathrm{GaP}$ y $\mathrm{SiC}$, se pueden apreciar en la Fig.[2] y la Fig.[3] respectivamente.

La estructura de las bandas de energía de ambos compuestos presentan una brecha indirecta de energía prohibida entre la banda de valencia y la banda de conducción, de $2.32 \mathrm{eV}$ para el GaP y de $2.22 \mathrm{eV}$ para el SiC, en ambos casos los cálculos LDA de la densidad de estados DOS, que se observa en la Fig.[4], Fig.[5] y Fig.[6], confirman la magnitud de la energía prohibida de estos sistemas cristalinos. Los valores están excelentemente de acuerdo a lo reportado por Y. Wang [1], C. S. Wang [10] para GaP y el reportado por L. Zhao [2] y a los resultados experimentales reportados en la literatura de especialidad.

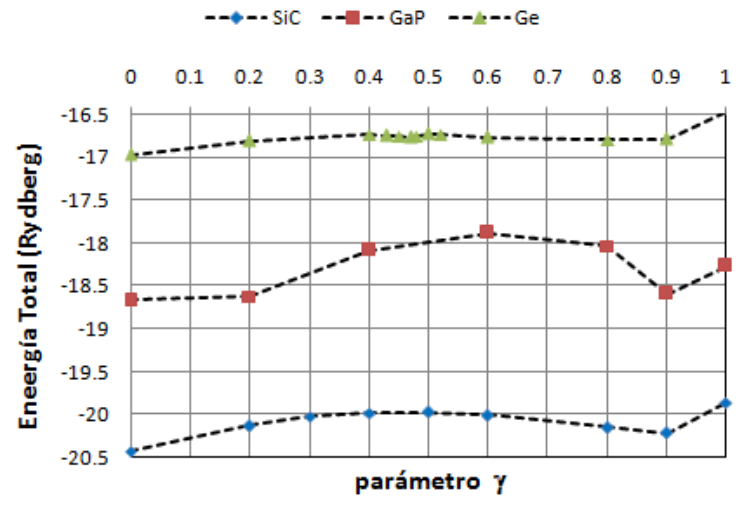

Figura 7: Energía total cálculadas para los cristales de Ge, GaP y $\mathrm{SiC}$

Los resultados del calculo LDA para la energía total, en función del parámetro de transferencia de carga a la red cristalina, de los sistemas de $\mathrm{Ge}, \mathrm{GaP}$ y $\mathrm{SiC}$ se presenta en la Fig.[7]. Para la curva de energía del Ge existe un mínimo relativo con un valor de energía de -16.77 Ry, cuando al parámetro, de transferencia de carga a la red cristalina, le corresponde justo el valor de 0.47 y es que para este valor se obtiene una buena estructura de las bandas de energía y de la densidad de estados para el sólido cristalino de Ge.

Para el caso de los cristales con estructura cristalina de tipo blenda de cinc,l como la de $\mathrm{GaP}$ y $\mathrm{SiC}$, resulta diferente a la del germanio, pues la energía total es mínima cuando el parámetro de transferencia de la carga a la red cristalina toma el valor 0.0 , en este caso la energía total toma el valor de -18.67 Ry para sistema cristalino de GaP y toma el valor de -20.43 Ry para el sistema cristalino de $\mathrm{SiC}$, tal como se aprecia en la Fig.[7]. 


\begin{tabular}{lr}
\hline Cristal & $R(a . u)$. \\
\hline $\mathrm{Ge}$ & 2.632 \\
$\mathrm{Ga}$ & 2.504 \\
$\mathrm{P}$ & 2.484 \\
$\mathrm{Si}$ & 2.527 \\
$\mathrm{C}$ & 1.660 \\
\hline
\end{tabular}

Tabla 1: Radio de las esferas atómicas en la red cristalina

\section{Conclusiones}

Los cálculos LDA hechos para las propiedades electrónicas usando la Density Functional Theory DFT y el método LMTO, dan excelentes resultados para el caso de los sistemas cristalinos con estructura cristalina de tipo zincblende, Gap y SiC. Para el caso de Ge los resultados muestran un excelente perfil de las bandas de energía y de la densidad de estados, pero con una banda de energía prohibida aproximadamente la mitad de $0.77 \mathrm{eV}$ reportado por Wang [10] en la literatura.

\section{Agradecimientos}

Se agradece a la UPG-FCF UNMSM, Mi sincero agradecimiento a la FIQ-UNAC por su apoyo financiero a la presente investigación.

\section{Referencias}

[1] Yin Wang; Electronic structure of III-V zinc-blende semiconductors from first principles, Physical Review B.87, 235203 (2013).

[2] G.L. Zhao y D. Bagayoko; Electronic Structure and charge transfer in $3 \mathrm{C}$ and $4 \mathrm{H}-\mathrm{SiC}$, New Journal of Physics.2, 16.1 (2000).

[3] H.L. Skriver; The Linear Muffin-Tin Orbitals method,
Danish natural science foundation, unpublished, Roskilde, july (1983).

[4] J.P. Perdew and S. Kurth; Density functionals nonrelativistic coulomb system in the new century, $C$. Fiolhais, F. Nogueira, M. Marques (Eds.):LNP,620, pp. 1-55, (2003).

[5] J.M. MacLaren; Parameterised local spin density exchange-correlation energies and potentials for electronic structure calculations, Computer Physics Communications.66, 383-391 (1991).

[6] C. Cabrera; Uso de la corrección de auto energía en el calculo de las propiedades electrónicas de sólidos cristalinos, tesis, Facultad de Ciencias Física, unmsm (1996).

[7] W. Kohn; Electronic structure of matter-wave functions and density functionals, Reviews of Modern Physics. vol.715, 1253 (1998).

[8] M.L. Cohen; Electronic structure and optical properties of semiconductors, Springer series in solid state sciences.75, 80 (1988).

[9] N.W. Ashcroft; Solid State Physics, Holt Rinehart and Winston, USA, 129 (1976).

[10] C.S.Wang; First principles electronic structure of Si, Ge, GaP, GaAs, ZnS and ZnSe., Physical Review B.24 6,3393 (1981). 